\title{
Surgical management of ankyloses of the temporomandibular joint by a piezoelectric device
}

\author{
Giuseppe Spinelli a, Domenico Valente ${ }^{\text {a }}$, Giuditta Mannelli ${ }^{\text {b }}$, Mirco Raffaini ${ }^{\text {a }}$, \\ Francesco Arcuri ${ }^{\text {a, * }}$ \\ a Unit of Maxillo-Facial Surgery, (Head: Chief Dr. Giuseppe Spinelli), Orthopedic Traumatological Center, Azienda Ospedaliera Universitaria Careggi, \\ Florence, Italy \\ ${ }^{\mathrm{b}}$ First Clinic of Otorhinolaryngology Head and Neck Surgery, University of Florence, Azienda Ospedaliera Universitaria Careggi, Florence, Italy
}

\section{A R T I C L E I N F O}

\section{Article history:}

Paper received 29 July 2016

Accepted 6 December 2016

Available online 18 December 2016

\section{Keywords:}

Piezosurgery

Temporomandibular joint ankylosis

Interpositional arthroplasty

\begin{abstract}
A B S T R A C T
Introduction: Piezosurgery is commonly used in different field of craniomaxillofacial surgery; since its introduction it has become one of the widely adopted technique for performing osteotomies in orthognathic surgery, distraction osteogenesis and dentoalveolar surgery. Little has been written regarding ultrasonic system for temporomandibular joint surgery (TMJ). In this prospective study we describe the use of piezoelectric device for ankylosis of the TMJ.

Materials and methods: We enrolled in this study 19 patients, 10 males and 9 females, mean (SD) age 24.6 (7.6) years, affected by unilateral ankylosis of TMJ who were surgically managed between January 2009 and December 2014 by interpositional arthroplasty with temporomyofascial muscle flap. We adopted in all cases a preauricular approach with temporal extension. The ankylotic block was removed with piezoelectric device in 9 patients and using traditional rotary bur in 10 cases. We investigated and compared the following parameters as surgical outcomes: intraoperative bleeding, duration of operation, incidence of infection, postoperative swelling and hematoma, mouth opening, nerve impairment and rate of recurrence.

Results: We noticed a substantial reduction in bleeding with the piezoelectric bone cutter when compared to traditional mechanical surgery (103 ml versus $117 \mathrm{ml}$; $\mathrm{p}<0.05$ ); however, we did not report any severe bleeding from the pterygoid plexus or maxillary artery. Operating time was longer in the piezo group (101 min versus $88 \mathrm{~min} ; \mathrm{p}<0.05$ ). There was a lower incidence of postoperative hematoma and swelling following piezoosteotomy. However, regarding postoperative nerve impairment and infection we did not observe any differences between the two groups. At one year follow-up mean (SD) mouth opening was $34(4.3) \mathrm{mm}$. We did not report recurrence of the disease.

Conclusion: Piezoelectric bone removal for the release of ankylosis of the TMJ is associated with minimal bleeding and few postoperative complications. We believe that piezosurgery allows surgeons to achieve better results compared to a traditional surgery. It is a possible alternative due to the clinical benefits demonstrated.
\end{abstract}

๑ 2016 European Association for Cranio-Maxillo-Facial Surgery. Published by Elsevier Ltd. All rights reserved.

\section{Introduction}

Piezosurgery is commonly used in different fields of craniomaxillofacial surgery. Since its introduction in dental practice (Lynn

\footnotetext{
* Corresponding author. Unit of Maxillo-Facial Surgery, Orthopedic Traumatological Center, Azienda Ospedaliera Universitaria Careggi, Largo Palagi 1, 50100, Florence, Italy. Fax: +39 0557949559.

E-mail address: fraarcuri@libero.it (F. Arcuri).
}

et al., 1942), it has become one of the most widely adopted techniques for performing osteotomies in orthognathic surgery, distraction osteogenesis, and dentoalveolar surgery. Currently, its use has spread to other surgical specialties, such as neurosurgery and otorhinolaryngology (Gleizal et al., 2007).

Piezosurgery uses ultrasonic vibrations to make clean and safe osteotomies. Piezoelectric devices have demonstrated better clinical results when compared with traditional instruments, including precision during the cut, reduced intraoperative blood loss, and 
lower incidences of postoperative nerve impairment, swelling, and hematoma (Beziat et al., 2007). Little has been written regarding ultrasonic systems for temporomandibular joint ankylosis (TMJa) (Landes et al., 2008; Olate et al., 2014).

TMJa is characterized by the formation of a fibrous, bony, or fibro-osseous tissue between the mandibular condyle and the base of the skull (glenoid fossa), which causes a limitation of movement of the jaw with an inability to open the mouth. Most of the time, TMJa is associated with disability of the following physiological functions: mastication, speech, swallowing, breathing, and nutrition. Moreover, it causes esthetic deformity because of disturbances of facial and mandibular growth. The degree of asymmetry (unilateral) or retrusion (bilateral) of the mandible depends on the time of onset and the duration of the disease (Guven, 2004).

TMJa occurs most frequently in the first and second decades of life. It is commonly associated with trauma, general or local infectious disease, rheumatic diseases (ankylosing spondylitis, rheumatoid arthritis), and previous TMJ surgery (He et al., 2008; Miyamoto et al., 2000).

According to the literature, there is controversy regarding the ideal surgical procedures for treating TMJa. However, the 3 surgical techniques most frequently used are joint reconstruction arthroplasty, interpositional arthroplasty, and gap arthroplasty. Operative protocols for TMJa can be one-stage or multistage, depending on the severity and complexity of the disease. The basic principles are resection of the ankylotic lesion, coronoidectomy, and early mobilization and aggressive postoperative physiotherapy (Kaban et al., 1990; Elgazzar et al., 2010; Guven, 2004).

Various materials have been used for reconstructive purposes at the gap of the TMJ, such as autologous (auricular cartilage, temporalis muscle, dermis fat, clavicular and costochondral bone grafts), alloplastic, and heterologous grafts. Finally, distraction osteogenesis, orthognathic surgery and esthetic surgery have been described after the treatment of the ankylosis to manage the facial deformity (Chossegros et al., 1997; Kearns, 1999; Gunsseeian, 1997; Guven, 2004).

In this prospective study, we describe the use of a piezoelectric device (Piezosurgery ${ }^{\mathbb{B}}$ ) for ankylosis of the TMJ, and we compare its use with the traditional high-speed drill (Stryker, USA).

\section{Materials and methods}

In this prospective randomized study, we enrolled all patients affected by ankylosis of the TMJ who were surgically managed by interpositional arthroplasty at the Unit of Maxillofacial Surgery of the Careggi Hospital and Meyer Children's Hospital of Florence. Informed written consent was obtained from all patients. Current standards of scientific research ethics were applied in the performance of this prospective study.

To be enrolled in this study, patients had to fulfill the following inclusion criteria: diagnosis with TMJa affecting the articular surfaces; available clinical and radiological data; postoperative followup period of at least 6 months; and unilateral involvement of the TMJ.

The exclusion criteria were: patients with systemic disease; treatment with surgical approaches and techniques other than interpositional arthroplasty (gap arthroplasty, reconstruction with costochondral graft and alloplastic reconstruction with hemijoint/ total joint prostheses); and insufficient preoperative or postoperative data.

The preoperative assessment included patient history (etiology of ankylosis), surgical data (affected side, mouth-opening measurements), and radiological evaluation (orthopantomography and computed tomography).
Patients were divided into 2 groups: Group A (removal of the ankylotic block by means of piezoelectric surgery), and Group B (management with high-speed drill and rotary burr).

\subsection{Surgical technique}

All of the procedures were performed by the same surgeon (G.S.) under general anesthesia with fibreoptic nasotracheal intubation. In all cases, a preauricular approach with temporal extension was adopted. After identification of the superficial temporal fascia, the dissection was carried out toward the periosteum of the zygomatic arch. Subsequently, a vertical incision of the joint capsule was done with the fascia retracted anteriorly to protect the facial nerve.

The ankylotic block was exposed and removed either with a piezoelectric device or with a rotary burr to create a gap of approximately $1.5 \mathrm{~cm}$. Bone cutting was performed from the lateral to the medial side of the TMJ perpendicular to the cortical surface together with ipsilateral intraoral coronoidectomy. Bony spicules were smoothed, and hemostasis was achieved by diathermy.

Care was taken to avoid the maxillary artery behind the medial aspect of the osseous mass. After resection of the ankylotic mass and creation of a space, an interpositional arthroplasty with temporomyofascial muscle flap was performed. The myofascial flap was turned outward over the zygomatic arch and placed downward between the glenoid fossa and the superior edge of the vertical ramus. It was sutured posteriorly and medially to the adjacent tissues with 4/0 non-resorbable suture. The wound was irrigated with saline solution, and a suction drain was inserted into the surgical wound and left in place until no more blood drained.

Postoperative antibiotics were prescribed for all patients to reduce the possibility of infection. Patients were discharged after satisfactory clinical evaluation. Professional physiotherapy (jaw exercises of mouth opening), massage, and deep heat therapy were prescribed after surgery.

Regular long-term follow-up was performed at an outpatient clinic at 1 week, 1 month, 3 months, 6 months, and then annually after surgery. Orthopantomography (OPG) and computed tomography (CT) were arranged at 6 months postoperatively (Figs. 1-4).

\subsection{Parameter assessment}

All evaluations were performed by the same clinician with the same parameters. Postoperative outcomes of TMJ surgery were investigated and compared based on the following assessment to address each device's advantages and disadvantages:

- Postoperative mouth interincisal opening: a caliber was adopted to measure the degree of mouth opening (interincisal distance) during the regular follow-up; it was compared to preoperative and intraoperative measurements;

- Intraoperative bleeding: blood loss was measured from the same type of suction instrument during each procedure;

- Length of the operation: duration of the procedure performed with the burr was compared with the length of the procedure performed with the piezoelectric device;

- Surgical site infection: this complication was defined as surgical wound/site infection by specific criteria (infection occurred within 30 days of the surgical operation with pain, swelling, and redness in association with purulent discharge from the surgical site and microbiological isolation from the wound culture); 

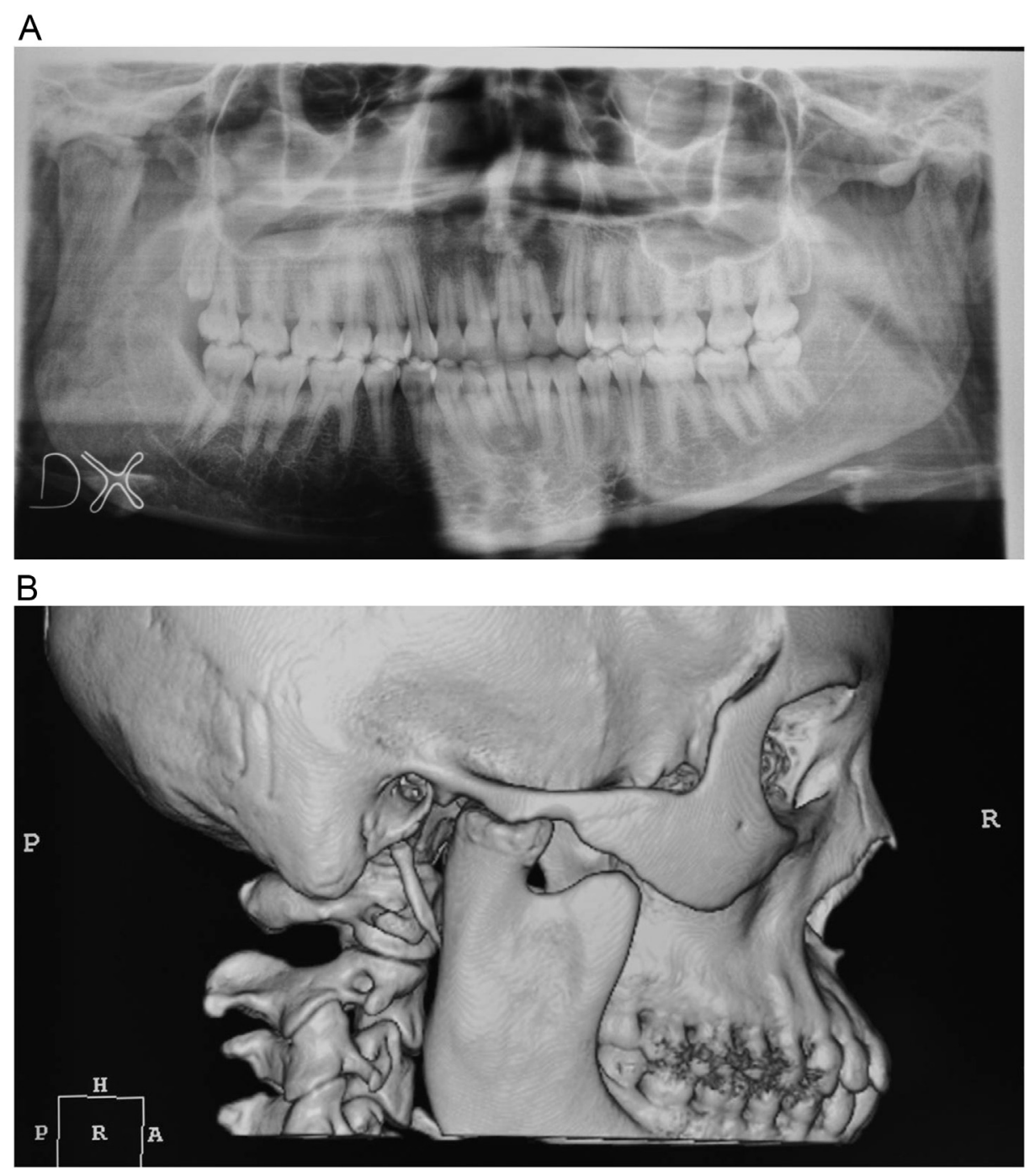

Fig. 1. (A, B) Preoperative orthopantomography (OPG) (A) and computed tomography (CT) scan (B) that show temporomandibular joint ankylosis (TMJa).

- Recurrence rate: relapse of the disease was evaluated after comparison between postoperative ( 1 year after surgery) and preoperative measurements;

- Facial nerve impairment: the House-Brackmann facial nerve grading system was used to assess any damage of the nerve at 1 month after surgery;

- Postoperative swelling and hematoma: these parameters were evaluated by comparing each side of the face at 1 month after surgery.

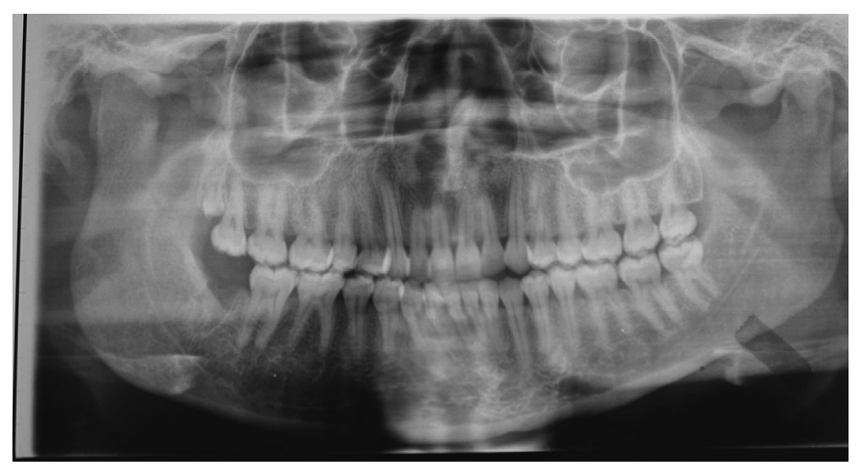

Fig. 2. Intraoperative detail of the arthroplasty using a piezoelectric device.

\subsection{Statistical analysis}

Standard descriptive statistics were used to summarize data. Comparison of clinical and biological characteristics between the 2

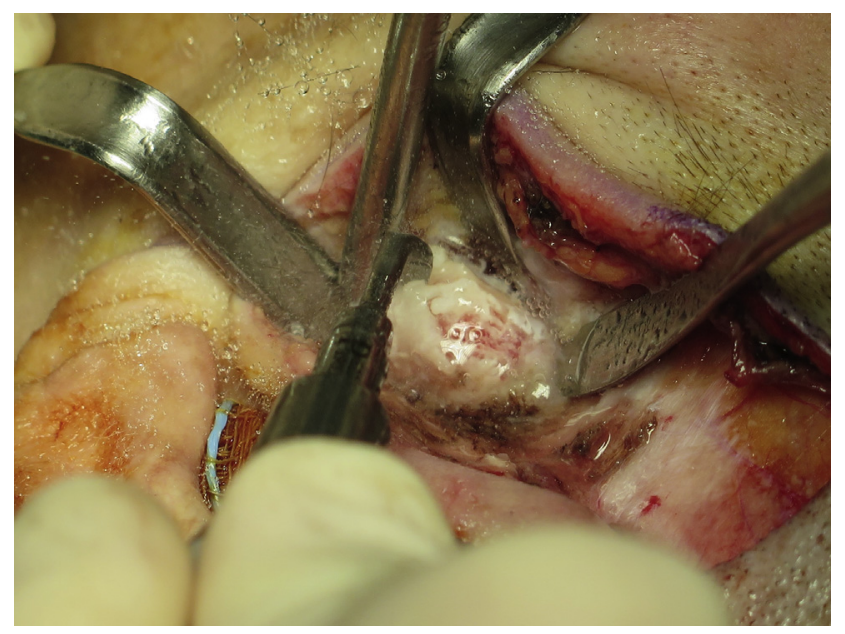

Fig. 3. Postoperative clinical view with improvement of the mouth opening. 


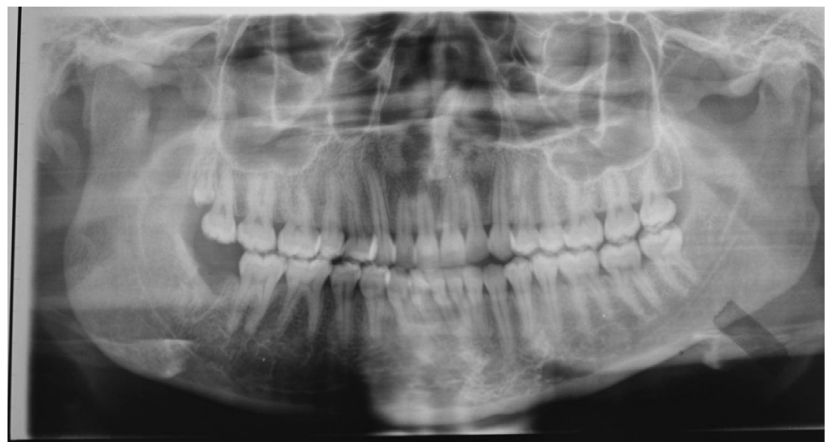

Fig. 4. Postoperative orthopantomography (OPG) scan which shows the correction of the temporomandibular joint ankylosis (TMJa) at 6 months after the surgical treatment.

groups of patients was performed by the Wilcoxon rank-sum test for continuous variables and Pearson Chi-square test (or Fisher exact test when appropriate) for categorical variables. Statistical analyses were performed using STATA (Stata Corporation, College Station, TX, USA). A 2-tailed p-value less than 0.05 was considered statistically significant.

\section{Results}

According to our inclusion criteria, of the 32 patients with ankylosis of the TMJ who underwent surgical treatment in our Unit between January 2009 and December 2014, only 19 were included in this investigation. Four patients were excluded due to the adoption of other surgical techniques (gap arthroplasty, joint reconstruction arthroplasty). Three patients were excluded because of bilateral involvement of the TMJ. Three patients were not included due to the lack of a long-term follow-up period. Two patients were excluded because of comorbidities. One patient had insufficient preoperative data.

The final study sample consisted of 19 patients ( 10 males and 9 females). The ages ranged from 15 to 39 years, and the mean age was 24.6 years. All cases were treated by interpositional arthroplasty with temporomyofascial muscle flap for monolateral ankylosis of the TMJ. Group A consisted of 9 cases (47.4\%) treated with piezoelectric device. Group B consisted of 10 patients (52.6\%) managed with traditional rotary burr. Each patient was investigated to assess any differences between the 2 procedures and consequences in terms of the final results.

The conventional burr was used on the right side in 5 patients (50\%) and on the left side in 5 patients (50\%); the piezoelectric instrument was used on the right side in 5 patients (55.6\%) and on the left side in 4 patients (44.4\%). Surgery was performed at a mean age of 24.6 years (standard deviation $[\mathrm{SD}]=7.6,95 \%$ confidence interval $[\mathrm{CI}]=20.9-28.2$, range $=12-39$ ). The mean follow-up was 16 months, ranging between 12 and 32 months.

Etiologic factors included trauma (13 cases, 68.4\%), unknown etiology (3 cases, $15.8 \%$ ), osteoarthritis ( 2 cases, $10.5 \%$ ), and previous TMJ surgery ( 1 case, $5.3 \%$ ). According to the classification of Sawhney (1986), 19 patients underwent TMJ surgery in different stages of ankylosis, based on computed tomography (CT) evaluation and confirmed after surgical exposure of the pathological joint. Three joints were classified as type II (15.8\%), 10 joints were classified as type III (52.6\%), and 6 joints were classified as type IV (31.6\%) (Table 1).

The mean preoperative mouth interincisal opening was $1.6 \mathrm{~mm}$ $(\mathrm{SD}=0.8,95 \% \mathrm{CI}=1.2-2.0$, range $=1-4)$. The mean intraoperative mouth opening was $40.3 \mathrm{~mm}(\mathrm{SD}=3.5,95 \% \mathrm{CI}=39-42$, range $=35-47)$. Postoperative mouth opening was measured 3
Table 1

Characteristics of the patients.

\begin{tabular}{llllll}
\hline $\begin{array}{l}\text { Patient } \\
\text { no. }\end{array}$ & Age & Sex & Etiology of the disease & Side & $\begin{array}{l}\text { Type of } \\
\text { ankylosis }\end{array}$ \\
\hline 1 & 24 & M & Trauma & Left & II \\
2 & 31 & F & Trauma & Right & III \\
3 & 15 & M & Trauma & Left & III \\
4 & 19 & F & Trauma & Right & III \\
5 & 12 & F & Previous TMJ surgery & Right & IV \\
6 & 34 & M & Osteoarthritis & Left & IV \\
7 & 31 & F & Unknown etiology & Right & III \\
8 & 23 & F & Trauma & Left & II \\
9 & 24 & M & Trauma & Left & III \\
10 & 26 & F & Trauma & Right & IV \\
11 & 17 & F & Trauma & Left & IV \\
12 & 19 & M & Osteoarthritis & Right & III \\
13 & 15 & F & Trauma & Left & II \\
14 & 39 & M & Trauma & Right & III \\
15 & 29 & M & Unknown etiology & Right & IV \\
16 & 19 & M & Trauma & Left & III \\
17 & 35 & F & Trauma & Right & IV \\
18 & 30 & M & Trauma & Right & III \\
19 & 25 & M & Unknown etiology & Left & III \\
\hline
\end{tabular}

times as follows: at 1 month (mean $=34.9 \mathrm{~mm}, \mathrm{SD}=4.1,95 \%$ $\mathrm{CI}=33-37$, range $=28-41$ ), at 6 months (mean $=34.4 \mathrm{~mm}$, $\mathrm{SD}=4.5,95 \% \mathrm{CI}=32-37$; range $=25-40)$, and at 1 year (mean $=33.9 \mathrm{~mm}, \mathrm{SD}=4.3,95 \% \mathrm{CI}=32-36$, range $=25$ ). We did not report relapse of the disease (interincisal mouth opening $<25 \mathrm{~mm}$ ) (Table 2).

Postoperatively, all patients demonstrated some degree of mandibular deviation toward the operated TMJ at maximum mouth opening. However, they achieved satisfactory occlusion. Regarding the differences between the 2 techniques, each patient was investigated to assess the final outcome. Surgical parameters included estimation of intraoperative bleeding during surgery (in milliliters $[\mathrm{ml}]$ ) and evaluation of the surgical procedure length (in minutes [min]).

The mean total blood loss for the piezoelectric group was $103 \mathrm{ml}$ $(\mathrm{SD}=23,95 \% \mathrm{CI}=85.1-120.0$, range $=76-132)$. The intraoperative bleeding with traditional surgery was $130 \mathrm{ml}$ ( $S D=27,95 \%$ $\mathrm{CI}=110.8-149.7$, range $=89-165)$. The mean length of the operation with a piezoelectric device was $101 \mathrm{~min}(\mathrm{SD}=13,95 \%$ $\mathrm{CI}=91-112$, range $=82-121)$. Conversely, the mean duration of the surgical procedure using a traditional burr was $88 \min (\mathrm{SD}=7$, $95 \% \mathrm{CI}=83-93$, range $=77-98)$. The mean overall hospital stay was 2.6 days (range $2-4$ days) (Table 3 ).

Table 2

Outcomes of the mouth interincisal opening.

\begin{tabular}{llllll}
\hline Patient no. & Preoperative & Intraoperative & 1 Month & 6 Months & 1 Year \\
\hline 1 & 1 & 41 & 35 & 36 & 35 \\
2 & 1 & 37 & 41 & 40 & 40 \\
3 & 1 & 43 & 29 & 29 & 28 \\
4 & 1 & 38 & 28 & 25 & 25 \\
5 & 2 & 43 & 33 & 32 & 32 \\
6 & 2 & 35 & 37 & 38 & 37 \\
7 & 1 & 42 & 38 & 37 & 36 \\
8 & 2 & 47 & 40 & 40 & 39 \\
9 & 1 & 42 & 37 & 35 & 36 \\
10 & 1 & 38 & 36 & 37 & 37 \\
11 & 2 & 35 & 35 & 34 & 33 \\
12 & 1 & 44 & 40 & 40 & 38 \\
13 & 2 & 40 & 38 & 37 & 37 \\
14 & 1 & 39 & 32 & 31 & 32 \\
15 & 4 & 38 & 34 & 36 & 36 \\
16 & 3 & 42 & 40 & 38 & 37 \\
17 & 1 & 36 & 30 & 29 & 29 \\
18 & 2 & 46 & 29 & 29 & 28 \\
19 & 1 & 41 & 32 & 30 & 30 \\
\hline
\end{tabular}


Table 3

Surgical data.

\begin{tabular}{lllll}
\hline $\begin{array}{l}\text { Patient } \\
\text { no. }\end{array}$ & $\begin{array}{l}\text { Type of mechanical } \\
\text { device }\end{array}$ & $\begin{array}{l}\text { Blood } \\
\text { loss }(\mathrm{ml})\end{array}$ & $\begin{array}{l}\text { Duration of } \\
\text { surgical } \\
\text { procedure }(\mathrm{min})\end{array}$ & $\begin{array}{l}\text { Hospital stay } \\
\text { (days) }\end{array}$ \\
\hline 1 & Piezosurgery & 129 & 101 & 2 \\
2 & Conventional saw & 145 & 79 & 3 \\
3 & Conventional saw & 123 & 91 & 3 \\
4 & Piezosurgery & 89 & 110 & 2 \\
5 & Conventional saw & 98 & 77 & 2 \\
6 & Conventional saw & 156 & 79 & 2 \\
7 & Piezosurgery & 78 & 98 & 3 \\
8 & Piezosurgery & 123 & 93 & 3 \\
9 & Conventional saw & 143 & 98 & 4 \\
10 & Piezosurgery & 119 & 82 & 2 \\
11 & Conventional saw & 98 & 91 & 3 \\
12 & Conventional saw & 165 & 95 & 3 \\
13 & Piezosurgery & 76 & 119 & 2 \\
14 & Piezosurgery & 90 & 101 & 3 \\
15 & Conventional saw & 132 & 87 & 4 \\
16 & Conventional saw & 89 & 94 & 2 \\
17 & Piezosurgery & 132 & 87 & 2 \\
18 & Conventional saw & 154 & 90 & 3 \\
19 & Piezosurgery & 87 & 121 & \\
\hline
\end{tabular}

We noticed a substantial reduction in bleeding with the piezoelectric bone cutter when compared to traditional mechanical surgery (102 ml versus $130 \mathrm{ml}, \mathrm{p}<0.05)$; however, we did not observe any severe bleeding from the pterygoid plexus or maxillary artery. Operating time was longer in the piezoelectric group (101 versus $88 \mathrm{~min}, \mathrm{p}<0.05$ ) (Table 4 ).

The piezoelectric ostectomy was easier and more precise when compared to the rotary saw method. We did not observe any damage to adjacent soft tissues. Bone exposure was also less extended during piezoelectric surgery; thus the risk of soft-tissue trauma was reduced.

We observed 1 case (10\%) of surgical site infection in Group B at 10 days after surgery, which resolved after 2 weeks of antibiotic treatment. Transient and mild facial nerve paresis (grade II) was observed in 2 cases ( 1 in Group A, 10\% and 1 in Group B, 11\%). Both cases resolved completely with physiotherapy at 6 months after surgery.

At 1 month after surgery, all patients were assessed for postoperative swelling and hematoma. Evidence of swelling was present in 4 of the 10 patients who underwent operation with the rotary burr (40\%) and in 1 patient treated with piezosurgery (11\%). Hematoma was still present in 1 patient treated with the burr (10\%). However, postoperative swelling and hematoma resolved completely after 6 months (Table 5).

There was a lower incidence of postoperative hematoma and swelling following piezo-osteotomy. However, regarding postoperative nerve impairment and infection, we did not observe any differences between the 2 groups.

The skin incisions healed without any visible scarring. Transient numbness of the surgical site occurred in 13 patients (68.4\%), and in all cases had resolved 6 months after surgery. The pain associated with the immediate aggressive physiotherapy decreased during the entirety of the study period.

Postoperative CT scans were performed on average 6.5 months ( $\mathrm{SD}=2.1$ months) after the procedures. The CT scan revealed the absence of reankylosis in all cases. None of the patients were lost to follow-up during the follow-up period.

\section{Discussion}

According to Sawhney's classification (Sawhney, 1986), ankylosis of the TMJ can be classified into 4 different types: type 1 (minimal bony fusion with extensive fibrous adhesions); type 2 (more bony tissue without fusion of the medial area); type 3 (bone between the mandible and the temporal bone); and type 4 (joint replaced entirely by bone). The basic principles for the treatment of TMJa are resection of the ankylotic block, placement of an interpositional graft, and intensive postoperative physiotherapy.

This study was a prospective evaluation of 19 patients affected by different stages of TMJa ( 10 joints with type III, 6 joints with type IV, and 3 joints with type II). We examined the efficacy of piezoelectric instruments in TMJa and compared its use with that of the traditional burr. The etiology of this disease includes trauma, infection, and previous TMJ surgery. Previous facial injury is the most frequent cause of this disease, which results in local hematoma and later ossification.

This study demonstrated a history of previous trauma in 13 (68\%) cases. This percentage is lower when compared to other findings, which range from $85 \%$ to $100 \%$ of the patients affected by TMJ ankylosis (Erol et al., 2006; Roychoudhury et al., 1999; Qudah et al., 2005).

According to the literature, maximal interincisal mouth opening in normal individuals is approximately $50 \mathrm{~mm}$; it is correlated with

Table 4

Surgical complications.

\begin{tabular}{|c|c|c|c|c|c|c|}
\hline $\begin{array}{l}\text { Patient } \\
\text { no. }\end{array}$ & $\begin{array}{l}\text { Type of mechanical } \\
\text { device }\end{array}$ & $\begin{array}{l}\text { Surgical site } \\
\text { infection }\end{array}$ & $\begin{array}{l}\text { Facial nerve } \\
\text { impairment }\end{array}$ & $\begin{array}{l}\text { Postoperative } \\
\text { swelling }\end{array}$ & $\begin{array}{l}\text { Postoperative } \\
\text { hematoma }\end{array}$ & Reankylosis \\
\hline 1 & Piezosurgery & No & No & No & No & No \\
\hline 2 & Conventional bur & No & No & No & No & No \\
\hline 3 & Conventional bur & No & No & No & No & No \\
\hline 4 & Piezosurgery & No & No & No & No & No \\
\hline 5 & Conventional bur & No & No & No & No & No \\
\hline 6 & Conventional bur & No & No & No & No & No \\
\hline 7 & Piezosurgery & No & Yes & No & No & No \\
\hline 8 & Piezosurgery & No & No & No & No & No \\
\hline 9 & Conventional bur & Yes & No & Yes & No & No \\
\hline 10 & Piezosurgery & No & No & No & No & No \\
\hline 11 & Conventional bur & No & No & No & No & No \\
\hline 12 & Conventional bur & No & Yes & Yes & No & No \\
\hline 13 & Piezosurgery & No & No & Yes & No & No \\
\hline 14 & Piezosurgery & No & No & No & No & No \\
\hline 15 & Conventional bur & No & No & Yes & No & No \\
\hline 16 & Conventional bur & No & No & Yes & Yes & No \\
\hline 17 & Piezosurgery & No & No & No & No & No \\
\hline 18 & Conventional bur & No & No & No & No & No \\
\hline 19 & Piezosurgery & No & No & No & No & No \\
\hline
\end{tabular}


Table 5

Analysis of patient data.

\begin{tabular}{|c|c|c|c|c|}
\hline Characteristic & Total $(\mathrm{N}=19)$ & Traditional surgery $(\mathrm{N}=10)$ & Piezosurgery $(\mathrm{N}=9)$ & p-value \\
\hline \multicolumn{5}{|l|}{ Age (years) } \\
\hline Mean (range) & $24.6(12-39)$ & $23(12-34)$ & $26(15-39)$ & 0.3463 \\
\hline SD & 7.6 & 7.6 & 7.6 & \\
\hline $95 \% \mathrm{CI}$ & $(20.9-28.2)$ & $(17.5-28.5)$ & $(20.5-32.1)$ & \\
\hline Etiology, n (\%) & & & & 0.582 \\
\hline Osteoarthritis & $2(10.5 \%)$ & $2(20.0 \%)$ & $0(0 \%)$ & \\
\hline Previous TMJ surgery & $1(5.3 \%)$ & $1(10.0 \%)$ & $0(0 \%)$ & \\
\hline Trauma & $13(68.4 \%)$ & $6(60.0 \%)$ & $7(77.8 \%)$ & \\
\hline Unknown & $3(15.8 \%)$ & $1(10.0 \%)$ & $2(22.2 \%)$ & \\
\hline Side, n (\%) & & & & 1.000 \\
\hline Left & $9(47.4 \%)$ & $5(50.0 \%)$ & $4(44.4 \%)$ & \\
\hline Right & $10(52.6 \%)$ & $5(50.0 \%)$ & $5(55.6 \%)$ & \\
\hline Type of ankyloses, n (\%) & & & & 0.187 \\
\hline II & $3(15.8 \%)$ & $0(0 \%)$ & $3(33.3 \%)$ & \\
\hline III & $10(52.6 \%)$ & $6(60.0 \%)$ & $4(44.4 \%)$ & \\
\hline IV & $6(31.6 \%)$ & $4(40.0 \%)$ & $2(22.2 \%)$ & \\
\hline \multicolumn{5}{|l|}{ Blood loss } \\
\hline Mean (range) & $117.1(76-165)$ & $130(89-165)$ & $103(76-132)$ & 0.0197 \\
\hline SD & 28 & 27 & 23 & \\
\hline $95 \% \mathrm{CI}$ & $(103.5-130.8)$ & (110.8-149.7) & $(85.1-120.0)$ & \\
\hline \multicolumn{5}{|l|}{ Duration } \\
\hline Mean (range) & $94(77-121)$ & $88(77-98)$ & $101(82-121)$ & 0.0220 \\
\hline SD & 12 & 7 & 13 & \\
\hline $95 \% \mathrm{CI}$ & $(88-110)$ & $(83-93)$ & $(91-112)$ & \\
\hline \multicolumn{5}{|l|}{ Preoperative } \\
\hline Mean (range) & $1.6(1-4)$ & $1.9(1-4)$ & $1.2(1-2)$ & 0.0783 \\
\hline SD & 0.8 & 1.0 & 0.4 & \\
\hline $95 \% \mathrm{CI}$ & $(1.2-2.0)$ & $(1.2-2.6)$ & $(0.9-1.6)$ & \\
\hline \multicolumn{5}{|l|}{ Intraoperative } \\
\hline Mean (range) & $40.3(35-47)$ & $40(35-46)$ & $40(36-47)$ & 0.6816 \\
\hline SD & 3.5 & 3.9 & 3.2 & \\
\hline $95 \% \mathrm{CI}$ & $(39-42)$ & $(38-43)$ & $(38-43)$ & \\
\hline \multicolumn{5}{|l|}{1 month } \\
\hline Mean (range) & $34.9(28-41)$ & $36(29-41)$ & $34(28-40)$ & 0.5387 \\
\hline SD & 4.1 & 4.3 & 4.1 & \\
\hline $95 \% \mathrm{CI}$ & $(33-37)$ & $(32-39)$ & $(31-37)$ & \\
\hline \multicolumn{5}{|l|}{6 months } \\
\hline Mean (range) & $34.4(25-40)$ & $35(29-40)$ & $34(25-40)$ & 0.5378 \\
\hline SD & 4.5 & 4.1 & 5.0 & \\
\hline $95 \% \mathrm{CI}$ & $(32-37)$ & $(32-38)$ & $(30-37)$ & \\
\hline \multicolumn{5}{|l|}{1 year } \\
\hline Mean (range) & $33.9(25-40)$ & $34(28-40)$ & $33(25-39)$ & 0.5930 \\
\hline SD & 4.3 & 4.1 & 4.6 & \\
\hline $95 \% \mathrm{CI}$ & $(32-36)$ & $(32-37)$ & $(30-37)$ & \\
\hline Surgical site infection, n (\%) & $1(5.3 \%)$ & $1(10 \%)$ & $0(0 \%)$ & 1.000 \\
\hline Facial nerve impairment, $\mathrm{n}(\%)$ & $2(10.5 \%)$ & $1(10 \%)$ & $1(11 \%)$ & 1.000 \\
\hline Postoperative swelling, n (\%) & $5(26.3)$ & $4(40 \%)$ & $1(11 \%)$ & 0.303 \\
\hline Postoperative hematoma, $\mathrm{n}(\%)$ & $1(5.3 \%)$ & $1(10 \%)$ & $0(0 \%)$ & 1.000 \\
\hline Reankylosis, n (\%) & $0(0 \%)$ & $0(0 \%)$ & $0(0 \%)$ & 1.000 \\
\hline At least one complication & $6(32 \%)$ & $4(40 \%)$ & $2(22 \%)$ & 0.628 \\
\hline
\end{tabular}

age, sex, and height (Ying et al., 2013). The recommended results after surgical treatment of TMJa in terms of postoperative mouth opening range between $30 \mathrm{~mm}$ and $35 \mathrm{~mm}$ (De Roo et al., 2016), which is in line with the results of our study.

Different surgical procedures have been described to correct TMJ ankyloses; however, there is no agreement regarding the ideal technique. Alloplastic materials can lead to infection and body reaction, and autogenous grafts are correlated with donor site morbidity. A recent meta-analysis described 4 treatment groups for TMJa (gap arthroplasty, interposition arthroplasty with temporalis muscle flap, reconstruction with costochondral graft, and alloplastic reconstruction). The authors reported that interpositional arthroplasty with temporalis muscle flap was better in terms of mouth opening when compared to gap arthroplasty and reconstruction with costochondral graft, and was better than gap arthroplasty in terms of recurrence rate (AlMoraissi et al., 2015).
Interpositional arthroplasty has become the preferred technique for management of TMJa (Dimitroulis, 2004) because of the less aggressive treatment and the interposition of tissue, which prevents reankylosis. However, reconstruction arthroplasty is still clinically indicated in complex cases with extensive ankylosis, bilateral disease, and facial deformity (El-Sayed, 2008).

Coronoidectomy has taken on a pivotal role in increasing interincisal mouth opening. The coronoid process tends to interfere with the medial aspect of the zygomatic arch and the posterior region of the zygomatic body, which results in restriction of the mouth opening. In our study, coronoidectomy was performed in all cases through an intraoral incision.

Another aspect is related to the degenerative changes of the masticatory muscles. Long periods of muscle inactivity lead to anatomical changes of the temporalis and masseter muscles, and this contributes to restriction of the mouth (Babu et al., 2013). Many authors have outlined the evidence that immediate mobilization and aggressive physiotherapy is the most successful strategy for 
TMJa (Asanami et al., 1990; Su-Gwan, 2001). We prefer to start physiotherapy 7 days after surgery to prevent adhesions and contractures of the soft tissue. It is our opinion that immediate mobilization can lead to bleeding and creation of a hematoma that may delay the healing process.

Regarding the interpositional graft, in all cases we used the temporomyofascial muscle flap, which was fixed to the adjacent soft tissue with non-resorbable suture.

Conventional methods of bone cutting with a rotary burr require wide exposure of the ankylotic mass, which can lead to increased postoperative pain and swelling of the surgical site. Moreover, it generates heat, which causes damage of the bone and peripheral osteonecrosis. Other disadvantages of traditional techniques are irregular osteotomy, increased bleeding, and mechanical vibrations (Beziat et al., 2007).

The main advantage of the relatively new technique of piezosurgery is its selectivity for mineralized structures. Thus, damage to soft tissues and vital structures is minimized, as well as bleeding from the osteotomy site. The vibration of the piezoelectric device is almost inappreciable to the surgeon. Instead, the microvibrations generate a precise cut and maintain a surgical site without blood with good intraoperative control. Increased surgical time and the cost of the device are 2 disadvantages of this technique (Olate et al., 2014; Jose et al., 2014).

In our study, 3 (15\%) postoperative complications occurred in 19 patients. They included 2 cases of facial nerve impairment ( 1 case for each group) and 1 case of surgical site infection (traditional surgery). All complications were managed conservatively. Facial nerve paresis resolved completely with physiotherapy at 6 months after surgery. Surgical site infection developed 10 days after surgery; it resolved after 2 weeks of antibiotic treatment after microbiological isolation from the wound culture. This report is lower when compared to that in a previous study (Saeed and Kent, 2003).

Intermaxillary fixation (IMF) with elastic therapy is clearly described in the scientific literature for TMJa patients (Matsuura et al., 2001). However, we never used this technique. In our opinion, it diminishes the results of professional physiotherapy and increases the risk of reankylosis.

In our study, dental occlusion remained unchanged after treatment except for some degree of mandibular deviation toward the operated TMJ. However, we did not need orthognathic surgery to correct the dental and skeletal deformities.

The mean length of the procedure performed by piezosurgery was longer when compared to the traditional method (101 min versus $88 \mathrm{~min} ; \mathrm{p}<0.05$ ) and was considered statistically significant. Regarding intraoperative bleeding, blood loss increased in the group treated by traditional surgery compared to the group treated by piezoelectric device ( $130 \mathrm{ml}$ versus $102 \mathrm{ml} ; \mathrm{p}<0.05$ ). Finally, at 1 month after surgery, evidence of swelling and hematoma was more common in the group treated with the rotary burr than in the piezoelectric group; however, this was not considered statistically significant.

In our cases, we reached maximal surgical precision with minimal damage to the soft tissues. The findings of our study are positive in terms of functionality, and recommend the use of interpositional arthroplasty with temporomyofascial muscle flap and piezoelectric device for TMJ ankylosis. However, the weaknesses of our study include the short follow-up period and the small sample size.

\section{Conclusion}

We conclude that our surgical protocol for a successful management of unilateral TMJa without facial deformity is the creation of a gap followed by interpositional arthroplasty with temporomyofascial muscle flap.

Moreover, our results suggest that the key element in the treatment of TMJa and the prevention of re-ankylosis is proper fixation of the muscle flap between the glenoid fossa and the superior edge of the vertical ramus, followed by aggressive postoperative physiotherapy.

In our experience, piezosurgery is a reliable method that ensures satisfying results in terms of surgical outcomes. Although the time of the procedure and the learning curve are lengthened by the use of the ultrasound device, it offers precise bone cutting while minimizing adjacent soft-tissue injury.

Piezoelectric bone removal for the release of ankylosis of the TMJ is associated with minimal bleeding and few postoperative complications. It is a possible alternative to traditional surgery due to the clinical benefits demonstrated.

\section{Conflict of interest}

The authors declare that there are no financial and personal relationships with other persons or organizations that could inappropriately influence this work.

\section{Financial disclosure}

None of the authors has financial conflicts or interests to report in association with the contents of this paper.

\section{References}

Al-Moraissi EA, El-Sharkawy TM, Mounair RM, El-Ghareeb TI: A systematic review and meta-analysis of the clinical outcomes for various surgical modalities in the management of temporomandibular joint ankylosis. Int J Oral Maxillofac Surg 44: 470-482, 2015

Asanami S, Kasazaki Y, Uchida I: Large exostosis of the mandibular coronoid process: report of a case. Oral Surg Oral Med Oral Pathol 69: 559-562, 1990

Babu L, Jain MK, Ramesh C, Vinayaka N: Is aggressive gap arthroplasty essential in the management of temporomandibular joint ankylosis? - a prospective clinical study of 15 cases. Br J Oral Maxillofac Surg 51: 473-478, 2013

Beziat JL, Vercellotti T, Gleiza A: What is piezosurgery? Two-years experience in craniomaxillofacial surgery. Rev Stomatol Chir Maxillofac 108: 101-117, 2007

Chossegros C, Guyot L, Cheynet F: Comparison of different materials for interposition arthroplasty in treatment of the temporomandibular joint ankylosis surgery: long term follow up in 25 cases. Br J Oral Maxillofac Surg 35: 157-160, 1997

De Roo N, Van Doorne L, Troch A, Vermeersch H, Brusselaers N: Quantifying the outcome of surgical treatment of temporomandibular joint ankylosis: a systematic review and meta-analysis. J Craniomaxillofac Surg 44: 6-15, 2016

Dimitroulis G: The interpositional dermis-fat graft in the management of temporomandibular joint ankyloses. Int J Oral Maxillofac Surg 33: 755-760, 2004

El-Sayed KM: Temporomandibular joint reconstruction with costochondral graft using modified approach. Int J Oral Maxillofac Surg 37: 897-902, 2008

Elgazzar RF, Abdelhady AI, Saad KA, Elshaal MA, Hussain MM, Abdelal SE, et al: Treatment modalities of TMJ ankylosis: experience in Delta Nile, Egypt. Int J Oral Maxillofac Surg 39: 333-342, 2010

Erol B, Tanrikulu R, Gorgun B: A clinical study on ankylosis of temporomandibular joint. J Craniomaxillofac Surg 34: 100-106, 2006

Gleizal A, Bera JC, Lavandier B, Beziat JL: Piezoelectric osteotomy: a new technique for bone surgery-advantages in craniofacial surgery. Childs Nerv Syst 23: 509-513, 2007

Gunsseeian R: Condylar reconstruction in extensive ankylosis of temporomandibular joint in adults using resected segment as autograft. Int J Oral Maxillofac Surg 26: 405-407, 1997

Guven O: Treatment of temporomandibular joint ankylosis by a modified fossa prosthesis. J Craniomaxillofac Surg 32: 236-242, 2004

He D, Ellis 3rd E, Zhang Y: Etiology of temporomandibular joint ankylosis secondary to condylar fractures: the role of concomitant mandibular fractures. J Oral Maxillofac Surg 66: 77-84, 2008

Jose A, Nagori SA, Virkhare A, Bhatt K, Bhutia O, Roychoudhury A: Piezoelectric osteoarthrectomy for management of ankylosis of the temporomandibular joint. Br J Oral Maxillofac Surg 52: 624-628, 2014

Kaban LB, Perrott DH, Fisher K: A protocol for management of temporomandibular joint ankylosis. J Oral Maxillofac Surg 48: 1145-1151, 1990

Kearns G: Subjective and objective assessment of the temporalis myofascial flap in previously operated temporomandibular joints. J Oral Maxillofac Surg 57: 1065-1067, 1999 
Landes CA, Stubinger S, Rieger J, Williger B, Ha TK, Sader R: Critical evaluation of piezoelectric osteotomy in orthognathic surgery: operative technique, blood loss, time requirement, nerve and vessel integrity. J Oral Maxillofac Surg 66: 657-674, 2008

Lynn JG, Zwemer RL, Chick AJ: The biological application of focused ultrasonic waves. Science 96: 119-120, 1942

Matsuura H, Miyamoto H, Ishimaru J, Kurita K, Goss AN: Effect of partial immobilization on reconstruction of ankylosis of the temporomandibular joint with an autogenous costochondral graft: an experimental study in sheep. Br J Ora Maxillofac Surg 39: 196-203, 2001

Miyamoto H, Kurita K, Ogi N, Ishimaru JI, Goss AN: The effect of an intraarticular bone fragment in the genesis of the temporomandibular joint ankylosis. Int J Oral Maxillofac Surg 29: 290-295, 2000

Olate S, Unibazo A, Almeida A, de Moraes M: Mandibular condylectomy revisited: technical notes concerning the use of an ultrasonic system. J Oral Maxillofac Surg 72: 481-484, 2014
Qudah M, Qudeimat MA, Al-Maaita: Treatment of TMJ ankylosis in Jordanian children-a comparison of two surgical techniques. J Craniomaxillofac Surg 33: 30-36, 2005

Roychoudhury A, Parkash H, Trikha A: Functional restoration by gap arthroplasty in temporomandibular joint ankylosis. A report of 50 cases. J Oral Surg Oral Med Oral Pathol Oral Radiol Endod 87: 166-169, 1999

Saeed NR, Kent JN: A retrospective study of the costochondral graft in TMJ reconstruction. Int J Oral Maxillofac Surg 32: 606-609, 2003

Sawhney CP: Bony ankylosis of the temporomandibular joint: follow-up of 70 patients with arthroplasty and acrylic spacer interposition. Plast Reconstr Surg 77: 29-40, 1986

Su-Gwan K: Treatment of temporomandibular joint ankylosis with temporalis muscle and fascia flap. Int J Oral Maxillofac Surg 30: 189-193, 2001

Ying QV, Bacic J, Abramowicz S, Sonis A: Cross sectional: normal maximal incisal opening and associations with physical variables in children. Pediatr Dent 35: 61-66, 2013 